\title{
Identifying Biomarkers in Lymph Node Metastases of Esophageal Adenocarcinoma for Tumor-Targeted Imaging
}

\author{
D. J. J. M. de Gouw ${ }^{1}$ - M. Rijpkema ${ }^{2}$. T. J. J. de Bitter ${ }^{3}$. V. M. Baart ${ }^{4}$ - C. F. M. Sier ${ }^{4}$ S. Hernot ${ }^{5}$. G. M. van Dam ${ }^{6}$. \\ I. D. Nagtegaal ${ }^{3}$ - B. R. Klarenbeek ${ }^{1}$ - C. Rosman ${ }^{1} \cdot$ R. S. van der Post ${ }^{3}$
}

Published online: 11 February 2020

(c) The Author(s) 2020

\begin{abstract}
Introduction Tumor-targeted imaging is a promising technique for the detection of lymph node metastases (LNM) and primary tumors. It remains unclear which biomarker is the most suitable target to distinguish malignant from healthy tissue in esophageal adenocarcinoma (EAC).

Objective We performed an immunohistochemistry study to identify viable tumor markers for tumor-targeted imaging of EAC.

Methods We used samples from 72 patients with EAC to determine the immunohistochemical expression of ten potential tumor biomarkers for EAC (carbonic anhydrase IX [CA-IX], carcinoembryonic antigen [CEA], hepatic growth factor receptor, epidermal growth factor receptor, epithelial membrane antigen [EMA], epithelial cell adhesion molecule [EpCAM], human epidermal growth factor receptor 2 [HER-2], urokinase plasminogen activator receptor, vascular endothelial growth factor-A [VEGF-A], and VEGF receptor 2). Immunohistochemistry was performed on tissue microarrays of LNM $(n=48)$, primary EACs $(n=62)$, fibrotic tissues $(n=11)$, nonmalignant lymph nodes $(n=24)$, and normal esophageal and gastric tissues $(n=40)$. Tumor marker staining was scored on intensity and percentage of positive cells.

Results EMA and EpCAM showed strong expression in LNM (>95\%) and primary EACs (>95\%). Significant expression was also observed for LNM and EAC using VEGF-A (85 and 92\%), CEA (68 and 54\%), and CA-IX (4 and 34\%). The other tumor biomarkers showed expression of 0-15\% for LNM and primary EAC. Except for VEGF-A, nonmalignant lymph node staining was scored as slight or absent.

Conclusions High expression rates and correlation between LNM in EAC combined with low expression rates in healthy lymph nodes and esophagus tissues were observed for EpCAM and CEA, meaning these are promising targets for tumortargeted imaging approaches for lymph nodes in patients with EAC.
\end{abstract}

\section{Key Points}

The use of intraoperative targeted imaging to detect lymph node metastases is promising and will be an important step forward in personalizing the surgical treatment of patients with esophageal cancer.

The most suitable biomarker for targeted imaging techniques in lymph node metastases of esophageal adenocarcinoma remains unclear.

Histological evaluation shows that epithelial cell adhe-

Electronic supplementary material The online version of this article (https://doi.org/10.1007/s40291-020-00448-9) contains supplementary material, which is available to authorized users. sion molecule and carcinoembryonic antigen are suitable targets for image-guided esophageal surgery, both before and after neoadjuvant chemoradiotherapy.

R. S. van der Post

Chella.vanderpost@ radboudumc.nl

Extended author information available on the last page of the article 


\section{Introduction}

The incidence of esophageal cancer has increased rapidly and is currently the eighth most common cancer worldwide [1]. This is mainly due to an increasing incidence of esophageal adenocarcinoma (EAC), which is among the fastest increasing incidences of all malignant tumors [2]. Surgical resection of EAC in combination with lymphadenectomy after neoadjuvant chemoradiotherapy (nCRT) is the gold standard of intentionally curative treatment in the Netherlands [3]. Surgical treatment is associated with severe morbidity, partly because of lymphadenectomy, and substantially affects quality of life [4]. Although lymph node metastases (LNM) are detected in up to $70 \%$ of patients at the time of diagnosis, this decreases to $31 \%$ after nCRT, suggesting that $69 \%$ of patients receive unnecessary lymphadenectomy [3]. Diagnostic techniques currently used in the management of esophageal cancer are insufficiently accurate to reliably predict LNM before surgery [5, 6]. An accurate imaging technique for diagnosing LNM and stratification of patients according to need for lymphadenectomy is urgently needed to personalize the surgical treatment of patients with esophageal cancer.

Tumor-targeted imaging is a promising technique for the detection of solid tumors and assessment of locoregional LNM. Several targeted imaging applications are used to stage cancer and LNM and to monitor response to nCRT $[7,8]$. Nuclear imaging techniques, such as single-photon emission computed tomography (SPECT)-CT or positron emission tomography (PET)-CT, can be used for preoperative staging of cancer and response monitoring [7, 9, 10]. Another rapidly emerging imaging field is intraoperative fluorescence imaging. Several clinical trials have investigated clinically available antibodies conjugated to a fluorophore for targeted fluorescence imaging of breast, kidney, colorectal, and head and neck cancers [8, 11-13]. The use of targeted intraoperative imaging to diagnose LNM and primary tumors on a submillimeter level holds great promise and can be used to support complete tumor resection in esophageal cancer [8].

The optimal target biomarker to visualize tumor cells using targeted imaging approaches remains unclear for esophageal cancer. Immunohistochemistry (IHC) of tumor tissue is a validated method of identifying the expression of potential biomarkers. The aim of this study was to use IHC to identify potentially viable tumor markers that would be suitable for imaging approaches for the detection of primary EAC, particularly LNM.

\section{Methods}

\subsection{Tissue Selection}

IHC was performed on normal and tumor tissue from 72 patients with EAC who underwent surgical resection in the Radboud University Medical Center between 2005 and 2018. Tissue samples of LNM in EAC $(n=48)$, primary EAC $(n=62)$, fibrotic tissue $(n=11)$, nonmalignant "healthy" lymph nodes $(n=24)$, and normal esophageal and gastric tissues $(n=40)$ were collected. Nonmalignant lymph nodes with or without features of total regression after nCRT (fibrotic tissue) served as validation for background signals in targeted imaging. This study was performed according to the requirements of the Medical Ethics Committee of the Radboud University Medical Center, registration number 2017-3759.

\subsection{Tumor Markers}

To identify the optimal tumor marker for intraoperative fluorescence imaging, we selected ten markers according to the availability of clinically used tracers for tumor-targeted imaging techniques and expression results gathered from the literature. Hepatic growth factor receptor (c-MET), carbonic anhydrase IX (CA-IX), epidermal growth factor receptor (EGFR), epithelial cell adhesion molecule (EpCAM), human epidermal growth factor receptor 2 (HER-2), carcinoembryonic antigen (CEA), epithelial membrane antigen (EMA; also known as MUC1), urokinase plasminogen activator receptor (uPAR), vascular endothelial growth factor A (VEGF-A) and one of its receptors (VEGFR2) were included.

\subsection{Immunohistochemistry}

Tissue microarrays (TMA) with cores of $2 \mathrm{~mm}$ were constructed from formalin-fixed paraffin-embedded archival specimens. The area of interest was marked on the original hematoxylin and eosin stained section. Cores from histological representative regions were selected and arranged in a new tissue array paraffin block. Per individual patient, at least two cores from every tissue were included in the TMA. Sections $(4 \mu \mathrm{m})$ were deparaffinized and rehydrated, and heat-induced epitope retrieval was conducted in $10 \mathrm{mM}$ sodium citrate buffer ( $\mathrm{pH} \mathrm{6.0)}$ or EDTA solution ( $\mathrm{pH} 9.0$ ) for 10 minutes in a microwave. No antigens were retrieved for CA-IX and VEGF-A. After blocking of endogenous peroxidase with $3 \% \mathrm{H}_{2} \mathrm{O}_{2}$, slides were incubated with primary antibodies. Visualization was carried out with a 1:2 dilution of Brightvision Poly-HRP anti$\mathrm{Ms} / \mathrm{Rb}$ immunoglobulin $\mathrm{G}$ in $0.05 \%$ phosphate-buffered 
saline-Tween, followed by a 7-min incubation at room temperature with BrightDAB (both from Immunologic, Duiven, the Netherlands). Slides were counterstained with hematoxylin. uPAR sections were treated similarly except that epitope retrieval was performed using PT link and a low-pH Envision FLEX target retrieval solution (Agilent, Santa Clara, CA, USA) and visualization was carried out with Envision anti-mouse (K4001, Agilent) and 3,3 diaminobenzidine tetrahydrochloride (Agilent). For HER-2, a ready-to-use HercepTest kit was used (Dako A/S, Denmark) according to the manufacturer's protocol. Appropriate positive controls were included for each antibody. Table 1 in the Electronic Supplementary Material (ESM) provides specific details of the primary antibodies used.

\subsection{Scoring System}

IHC scoring was performed by the researcher (DG) and gastrointestinal pathologist (RP). Validation of IHC scoring was independently performed by a second gastrointestinal pathologist (IN) in a sample of 30 randomly selected TMA cores for each staining. Tumor marker expression was scored on intensity (none $=0$, slight $=1$, moderate $=2$, strong $=3$ ) and percentage of positive tumor cells (estimation). All markers were scored on membranous expression, except for VEGF-A and VEGFR2, which were scored on cytoplasmic expression. Examination results for duplicate cores were consolidated into one score, where the highest staining was scored when the intensity varied between cores within a patient. The threshold for a positive detection rate was defined as an intensity of $\geq 2$ for $>10 \%$ of the cells. If all primary tumor or LNM cores were missing from a patient, the set was defined as missing and excluded from analysis.

To discriminate between tumor and background tissue by tumor-targeted imaging, the tumor-to-normal $(T / N)$ ratio was calculated between the primary tumor and normal esophageal tissue and for metastatic and normal lymph nodes. The $T / N$ ratio was defined as the average total immunostaining score (TIS) of tumor tissue divided by the average TIS score for healthy tissue. The TIS score was calculated per region of interest by multiplying the mean intensity by the mean percentage of positive cells using five categories $(0=$ none; $1=<25 \% ; 2=25-49 \% ; 3=50-74 \% ; 4=>75 \%$ ).

\subsection{Statistical Analysis}

Statistical analyses were performed using $\mathrm{R}$ (version 3.2.1). The interobserver variation between pathologists was calculated with kappa statistics, and the Spearman rank correlation test was performed to test for associations with clinical parameters. In all tests, a $p$ value $<0.05$ was considered significant.
Table 1 Patient characteristics

\begin{tabular}{|c|c|}
\hline Characteristics & All $(n=72)$ \\
\hline Age at surgery, years & $64(44-82)$ \\
\hline \multicolumn{2}{|l|}{ Subtype } \\
\hline Intestinal & $63(87.5)$ \\
\hline Diffuse & $7(9.7)$ \\
\hline Other & $2(2.8)$ \\
\hline Tumor size $(\mathrm{cm})$ & $3(0.2-9.0)$ \\
\hline \multicolumn{2}{|l|}{ T-category } \\
\hline (y)pT0 & $6(8.3)$ \\
\hline (y)pT1 & $12(16.7)$ \\
\hline (y)pT2 & $25(34.7)$ \\
\hline (y)pT3 & $25(34.7)$ \\
\hline (y)pT4 & $1(1.4)$ \\
\hline Unknown & $3(4.2)$ \\
\hline \multicolumn{2}{|l|}{ N-category } \\
\hline (y)pN0 & $16(22.2)$ \\
\hline (y)pN1 & $54(75.0)$ \\
\hline Unknown & $2(2.8)$ \\
\hline Number of dissected lymph nodes & $14(2-28)$ \\
\hline Lymph node metastases & $3(1-20)$ \\
\hline \multicolumn{2}{|l|}{ Neoadjuvant therapy } \\
\hline None & $23(31.9)$ \\
\hline nCRT (CROSS) & $49(68.1)$ \\
\hline \multicolumn{2}{|l|}{ Mandard grade } \\
\hline TRG 1 & $3(6.1)$ \\
\hline TRG 2 & $14(28.6)$ \\
\hline TRG 3 & $19(38.8)$ \\
\hline TRG 4 & $8(16.3)$ \\
\hline TRG 5 & $5(10.2)$ \\
\hline
\end{tabular}

Data are presented as median (range) or $n(\%)$ unless otherwise indicated

CROSS scheme used in the CROSS study [3] (this scheme includes intravenous carboplatin and intravenous paclitaxel with concurrent radiotherapy followed by surgery), Mandard grade response to neoadjuvant therapy according to Mandard classification, $n$ number of patients, $\mathrm{N}$-category $\mathrm{N}$ stadium according to 8th TNM staging [30], $n C R T$ neoadjuvant chemoradiotherapy, T-category $\mathrm{T}$ stadium according to 8th TNM staging [30], TNM tumor, node, metastases, TRG1 complete response, $T R G 2$ presence of rare residual cancer, $T R G 3$ increase in the number of residual cancer cells, but predominantly fibrosis, TRG4 residual cancer outgrowing fibrosis, TRG5 absence of regressive changes [31]

\section{Results}

\subsection{Patient Characteristics}

Table 1 summarizes the characteristics of the patients with EAC included in this study. In total, 72 patients were included, of whom 49 had received pretreatment with nCRT; 54 patients with LNM (75\%) were included. Of 
these, 45 patients had sufficient data to compare expression from LNM with that from the corresponding primary tumor for all tumor markers.

\subsection{Expression of Esophageal Adenocarcinoma Markers}

Figure 1 provides an overview of the expression of each individual tumor marker. EMA and EpCAM were 100\% expressed in LNM and $98 \%$ in primary EAC, with a median intensity of 3 (range 2-3). VEGF-A, CEA and CA-IX expression was observed in 41 of 48 (85\%), 33 of $48(68 \%)$, and 2 of 48 (4\%) LNM and in 57 of $62(92 \%)$, 35 of $62(54 \%)$, and 21 of 62 (34\%) primary EACs, respectively. The expression of other tumor biomarkers was low and ranged between 0 and $15 \%$ for both LNM and primary EAC (Figs. 1, 2). The correlation of the expression patterns between EAC and LNM is shown in Table 2 in the ESM and summarized in Table 2. An agreement of 100\% between expression in LNM and the corresponding primary EAC was found for EMA and EpCAM.

\subsection{Expression of Tumor Markers in (Sub)Total Response}

To find suitable targets to monitor treatment response after nCRT, expression in minimal residual tumor and fibrotic tissue is important. Only VEGF-A expression was observed in the fibrotic tissue of patients with complete response to
nCRT (Mandard classification 1). In patients with a subtotal response (Mandard 2), expression rates of $100 \%$ were observed in LNM and residual tumor tissue for EpCAM and EMA. The expression rates of CA-IX, CEA, c-MET, HER-2, and VEGF-A were $0 \%, 78 \%, 11 \%, 11 \%$, and $67 \%$ in LNM and $36 \%, 45 \%, 0 \%, 10 \%$, and $100 \%$ in residual EAC of patients with subtotal responses, respectively. EGFR, uPAR, and VEGFR2 showed no expression in subtotal responses to nCRT (Fig. 1 in the ESM).

\subsection{Expression of Tumor Markers in Nonmalignant Tissue}

Staining in nonmalignant "healthy" and fibrotic lymph nodes was slight or absent for most markers, except for VEGF-A, where extracellular expression was strong (Fig. 3, supplemental Fig. 2). For EMA, uPAR, and VEGFR2, background staining was slight in normal lymph nodes. Staining for both VEGF-A and EMA was strong in nonmalignant squamous esophageal and gastric mucosal tissue. Background expression was slight for CEA and EpCAM in nonmalignant esophageal tissue, and without expression in normal lymph nodes. For CA-IX, expression was moderate to strong in normal muscle cells.

\subsection{Target Selection}

Table 2 shows the most important findings for target selection. To select suitable tumor markers, a high $T / N$ ratio is

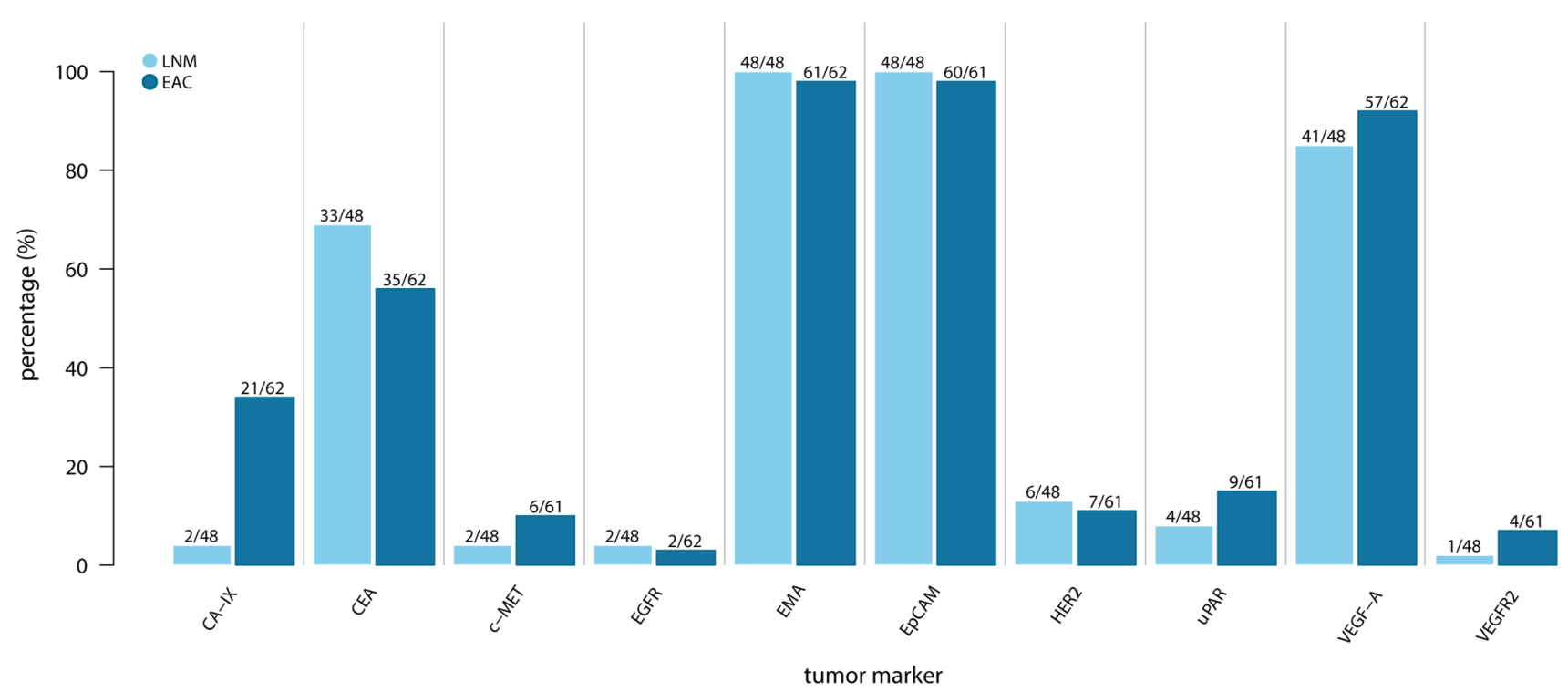

Fig. 1 Tumor marker expression in lymph node metastases and primary esophageal adenocarcinoma. CA-IX carbonic anhydrase IX, $C E A$ carcinoembryonic antigen, $c-M E T$ hepatic growth factor receptor, $E A C$ esophageal adenocarcinoma, EGFR epidermal growth factor receptor, EMA epithelial membrane antigen, EpCAM epithelial cell

adhesion molecule, HER-2 human epidermal growth factor receptor 2, $L N M$ lymph node metastases, $U P A R$ urokinase plasminogen activator receptor, $V E G F-A$ vascular endothelial growth factor-A, VEGFR2 VEGF receptor 2 


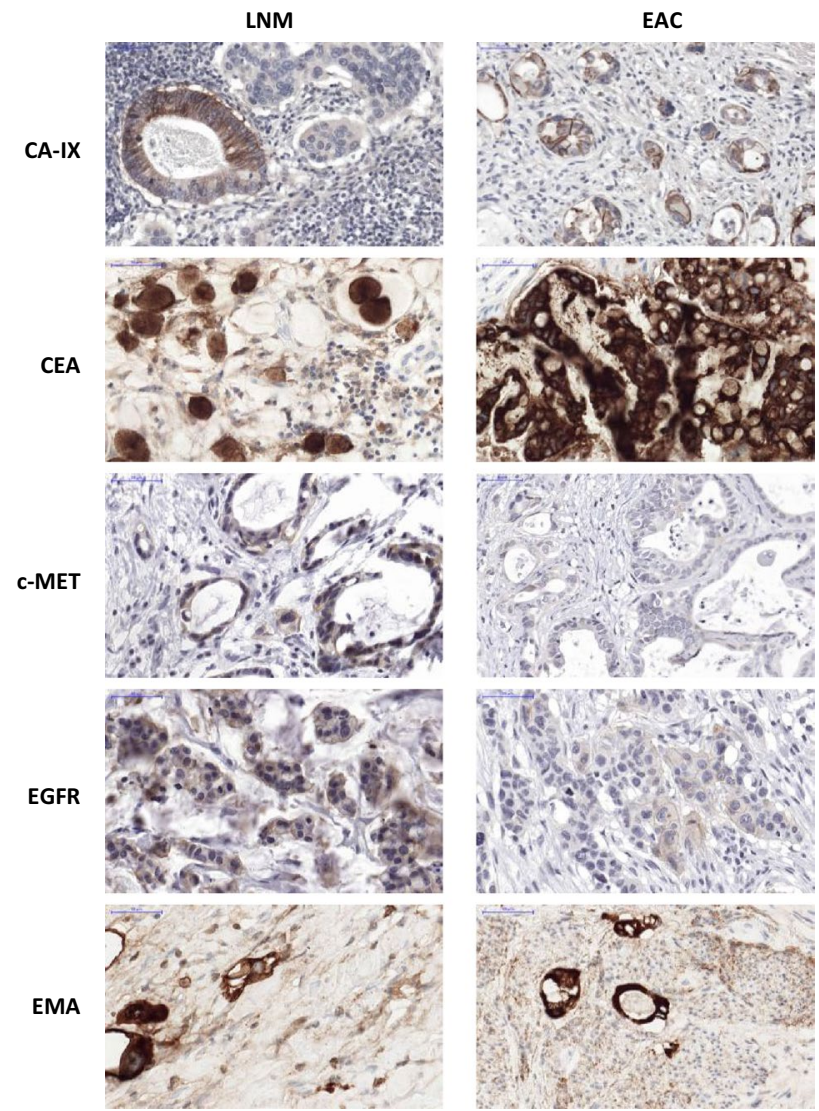

Fig. 2 Example of immunohistochemical staining of a metastatic lymph node and corresponding primary esophageal adenocarcinoma for each tumor marker. Scalebar equals $50 \mu \mathrm{m}$. $C A-I X$ carbonic anhydrase IX, CEA carcinoembryonic antigen, $c-M E T$ hepatic growth factor receptor, $E A C$ esophageal adenocarcinoma, $E G F R$ epidermal

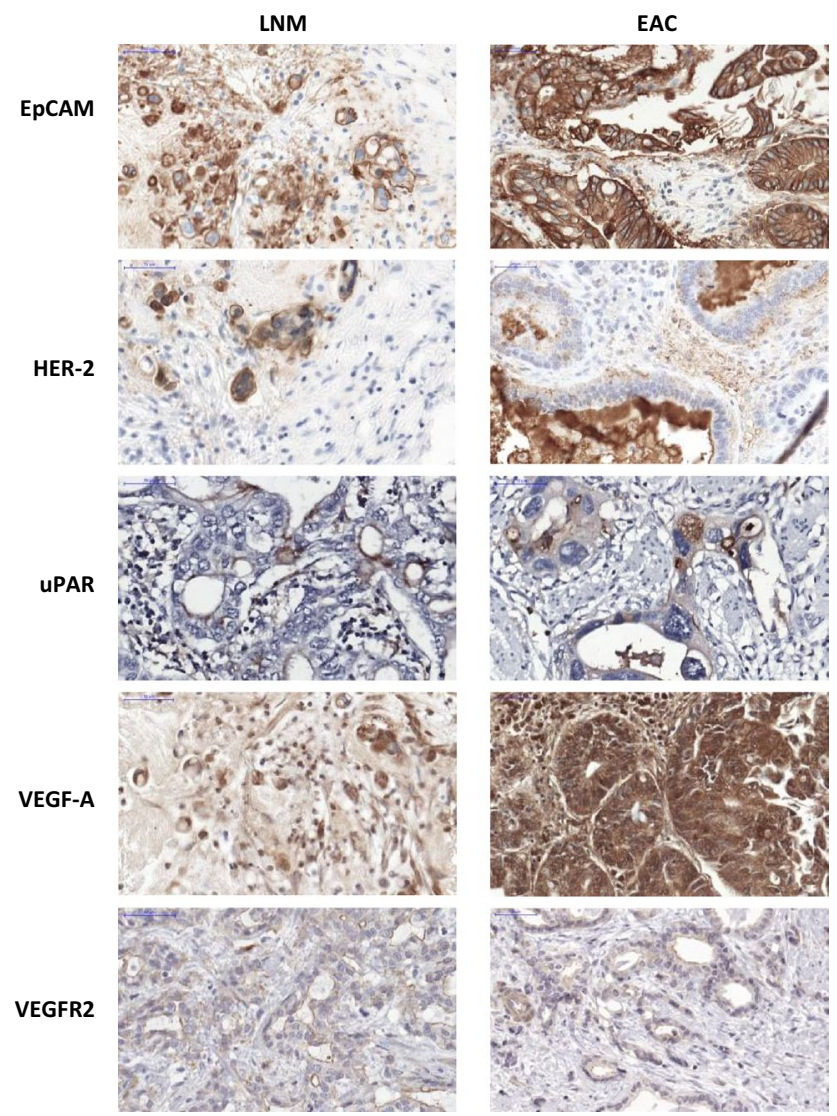

growth factor receptor, EMA epithelial membrane antigen, EpCAM epithelial cell adhesion molecule, HER-2 human epidermal growth factor receptor-2, $L N M$ lymph node metastases, $U P A R$ urokinase plasminogen activator receptor, $V E G F-1$ vascular endothelial growth factor-1, VEGFR2 VEGF receptor-2

Table 2 Characteristics of tumor markers for targeted imaging

\begin{tabular}{lcccccc}
\hline Target & $\begin{array}{c}\text { \% with positive } \\
\text { LNM expression }\end{array}$ & $\begin{array}{l}\text { \% with positive } \\
\text { EAC expression }\end{array}$ & $T / N$ ratio LNM & $T / N$ ratio EAC & $\begin{array}{c}\text { Agreement EAC and } \\
\text { LNM expression }\end{array}$ & Previously targeted imaging \\
\hline CA-IX & 4 & 34 & $>10$ & $>10$ & 65 & Clinical study [13] \\
CEA & 68 & 54 & $>10$ & 3.62 & 72 & Clinical study [27] \\
C-MET & 4 & 10 & $>10$ & $>10$ & 87 & Clinical study [32] \\
EGFR & 4 & 3 & $>10$ & 1.00 & 96 & Clinical study [33] \\
EMA & 100 & 98 & $>10$ & $>10$ & 100 & Clinical study [34] \\
EPCAM & 100 & 98 & $>10$ & $>10$ & 84 & Preclinical [29] \\
HER-2 & 13 & 11 & 1.09 & $>10$ & 89 & Clinical study [35] \\
uPAR & 8 & 15 & 1.21 & 0.91 & 80 & Clinical study [36] \\
VEGF-A & 85 & 92 & 0.42 & 0.33 & 93 & Clinical study [12] \\
VEGFR2 & 2 & 7 & & & Preclinical [37] \\
\hline
\end{tabular}

$C A-I X$ carbonic anhydrase IX, CEA carcinoembryonic antigen, $c$-MET hepatic growth factor receptor, EAC esophageal adenocarcinoma, $E G F R$ epidermal growth factor receptor, EMA epithelial membrane antigen, EpCAM epithelial cell adhesion molecule, HER-2 human epidermal growth factor receptor 2, LNM lymph node metastases, T/N ratio EAC tumor to background esophageal adenocarcinoma and nonmalignant esophageal tissue, $T / N$ ratio $L N M$ tumor to background ratio lymph node metastases and nonmalignant lymph nodes, $u P A R$ urokinase plasminogen activator receptor, VEGF-A vascular endothelial growth factor-A, VEGFR2 VEGF receptor 2 


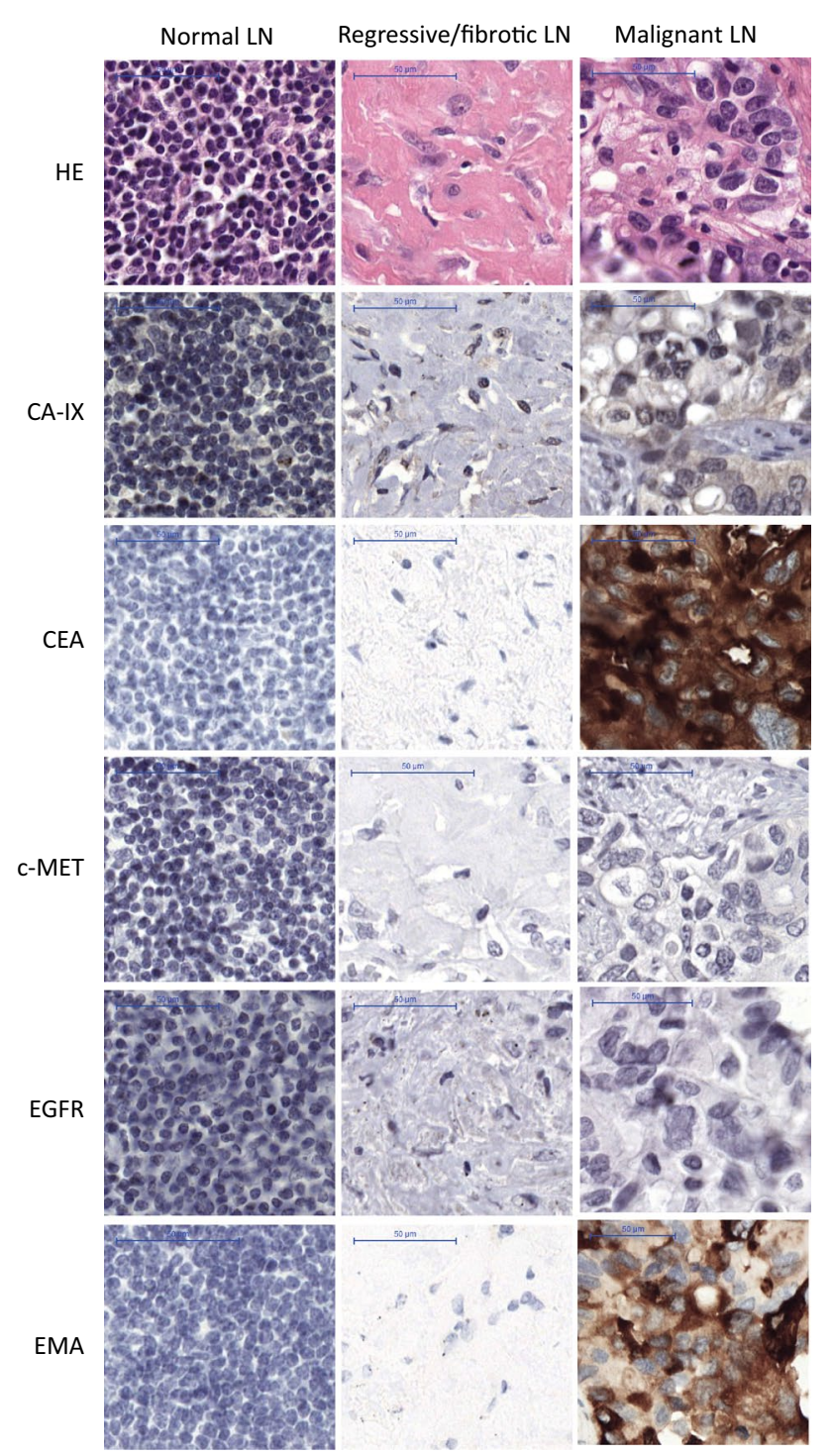

Fig. 3 Example of immunohistochemical staining of one normal (left), one regressive (middle) and one malignant (right) lymph node. Each of the three lymph nodes (normal, regressive, malignant) is selected from the same patient to show an example of the staining pattern of the various tumor markers. Scalebar equals $50 \mu \mathrm{m}$. $C A$ $I X$ carbonic anhydrase IX, CEA carcinoembryonic antigen, $c-M E T$

important to distinguish tumor tissue from nonmalignant tissue. With respect to LNM, a low $T / N$ was found for UPAR, VEGF-A, and VEGFR2, which suggests it is hard to distinguish LNM from nonmalignant lymph nodes. For the other markers, a high $T / N$ ratio of $>10$ was found, which is suitable for targeted imaging approaches (Fig. 2 in the ESM). The $T / N$ ratio for primary EAC and healthy esophageal tissue was one or less for VEGF-A, VEGFR2, and EMA because the TIS values were equal in tumor tissue and healthy tissue. A moderate $T / N$ ratio of 3.63 was found for CEA, and the

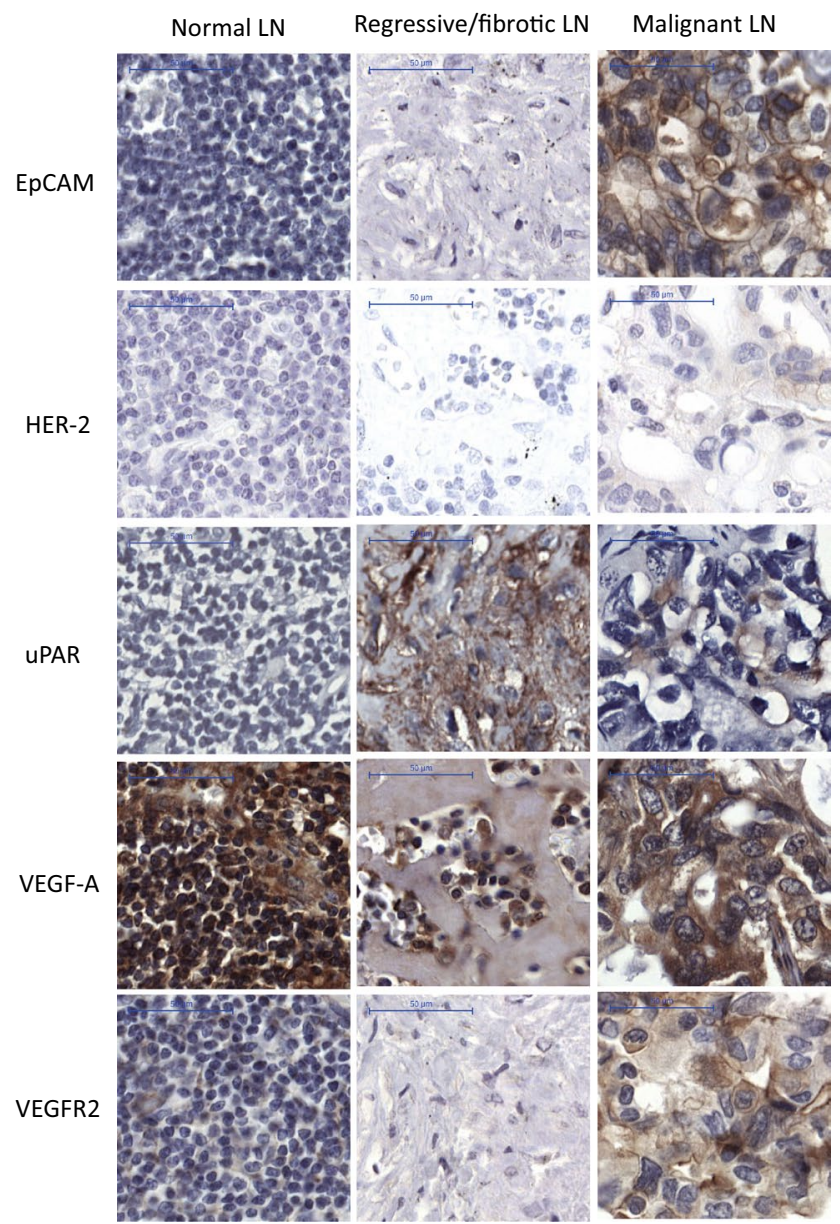

hepatic growth factor receptor, $E A C$ esophageal adenocarcinoma, $E G F R$ epidermal growth factor receptor, $E M A$ epithelial membrane antigen, EpCAM epithelial cell adhesion molecule, $H E$ hematoxylin and eosin, HER-2 human epidermal growth factor receptor-2, $L N$ lymph node, $u P A R$ urokinase plasminogen activator receptor, $V E G F$ 1 vascular endothelial growth factor-1, VEGFR2 VEGF receptor-2

ratio was $>10$ for the other markers. Furthermore, the candidate tumor markers (CEA, EMA, EpCAM) were investigated in a larger set of normal and tumor esophageal tissues using GEPIA [14], a web-based tool for gene expression profiling based on The Cancer Genome Atlas (TCGA) data. Only EpCAM showed significantly higher expression in tumor than in normal tissue (LOG2-fold change 4.6, $p<0.01$ ) (Fig. 3 in the ESM). Table 3 in the ESM summarizes the function, expression pattern, and expression levels in EAC based on the literature. 


\subsection{Subgroup Analysis}

Figure 1 and Table 4, both in the ESM, show the results of the subgroup analysis performed for neoadjuvant treatment. No clinically relevant difference was found in expression between patients receiving and not receiving nCRT.

\subsection{Validation of Immunohistochemistry Scoring}

The agreement between the IHC scoring of both pathologists was $94 \%$, with a corresponding kappa value for interobserver agreement of $0.88(p<0.001)$.

\section{Discussion}

We studied the expression of ten tumor markers to provide the first step toward clinical implementation of tumor-targeted imaging for LNM and EAC. High expression rates in LNM and corresponding primary EAC were observed using immunohistochemical antibodies for EpCAM, EMA, VEGF$\mathrm{A}$, and CEA. Expression in nonmalignant lymph nodes was only slight for all except VEGF-A. However, a low $T / N$ ratio was found for EAM in healthy esophageal and gastric tissue, which indicates that it may be hard to distinguish tumor from background tissue using targeted imaging approaches. Therefore, EpCAM seems the most promising target for targeted imaging of LNM in EAC since a strong expression of CEA is less frequent than that of EpCAM. Nonetheless, an imaging marker might show different kinetics in tumor cells than in nonmalignant tissue [15]. Furthermore, since expression did not differ between patients receiving and not receiving nCRT, and most markers showed a high overall correlation of up to $100 \%$ between immunohistochemical expression in LNM and primary EAC, it is possible to predict marker expression in LNM using IHC of the biopsy before treatment. Therefore, the most suitable marker can be selected for each individual patient before surgery, which also means CA-IX and UPAR are promising targets in a select subset of patients.

IHC results for numerous tumor markers in EAC have been described previously, mostly in terms of therapy options rather than imaging. Driessen et al. [16] studied the IHC expression of CA-IX and VEGF-A in 39 patients with EAC and found that CA-IX expression (49 vs. 34\%) was similar to that in our results but that VEGF-A expression was lower (69 vs. 92\%). However, another study reported a higher VEGF expression rate in tumor and background tissue, as corroborated by our results [17]. Chan et al. [18] studied the IHC expression of EGFR, HER-2, and c-MET in patients with EAC and reported a higher expression of these markers than that in our results; however, this study included only patients with early disease and primary surgery. HER-2 has been often studied in esophageal cancer for treatment with trastuzumab [18]. A systematic review of nine studies reported a pooled expression of 25\% (146 of 573 patients) for HER-2 in EAC, but this meta-analysis revealed large differences in results between studies [19]. The pooled expression of uPAR was reported as 57\% (range 14-90) for 12 gastroesophageal cancer studies [20], which is considerably higher than the $15 \%$ in our results. This may be explained by the random selection of tumor tissue. Generally, uPAR expression is higher at the invasive front of the tumor and lower at the core [21]. Reported IHC expression rates of CEA, EMA, and EpCAM are comparable to those in our results [22-25].

The current study provides a detailed overview of the expression of ten biomarkers in LNM, the corresponding primary EAC, fibrotic tissue after treatment, and surrounding normal tissue. The main strength of the study is that we investigated not only biomarker expression on LNM and EAC but also the effect of neoadjuvant treatment on that expression. Some potential limitations should also be discussed. First, biomarker selection was based on the literature and the availability of imaging agents for promising clinical translation, so other potential EAC biomarkers were not included. Second, the antibodies used in this study differ from those that will be used for targeted imaging. Therefore, the extent to which the IHC results are comparable with results of in vivo imaging remains unclear. Third, the same threshold for marker positivity is used in all tumor markers to translate the results to promising markers for targeted imaging. Finally, IHC scoring is not an objective measurement, so results should be considered semi-quantitatively [26]. However, the interobserver variability between the pathologists was low.

Image-guided surgery using fluorescently labeled tracers to target specific tumor markers and detect LNM intraoperatively is a promising application for targeted imaging. During surgery, extensive lymphadenectomy could be omitted or minimized in patients with limited or no LNM, thereby reducing the associated morbidity [4]. To our knowledge, tumor-targeted imaging has never been studied in patients with EAC to detect LNM during surgery, but a suitable tumor marker might enable this. EpCAM and CEA seem of particular interest for clinical translation given our current findings. Recently, a CEA-targeted fluorescent probe for intraoperative imaging, SGM-101, was validated in 26 patients with colorectal cancer [27] and 12 patients with pancreatic cancer [28]. Results indicated that the probe was safe and feasible for visualizing both primary adenocarcinomas and small metastases. A fluorescent tracer targeting EpCAM has not yet been used in human clinical trials. However, Boogerd et al. [29] reported on a fluorescent antiEpCAM tracer that was tested preclinically. Further work should focus on the clinical validation of EpCAM and CEA 
targets for intraoperative tumor-targeted imaging in EAC to personalize esophageal surgery.

\section{Conclusion}

High expression rates in LNM and corresponding primary EAC were observed using immunohistochemical antibodies for CEA, EpCAM, EMA, and VEGF-A. For EpCAM and CEA, expression was only slight in nonmalignant esophageal tissue, meaning these are promising viable EAC tumor markers for tumor-targeted imaging. Further studies should be conducted to determine the clinical value of these markers for tumor-targeted imaging approaches.

Acknowledgements The authors thank Kiek Verrijp, Hans Peters, and Chayenne van Duijn for technical assistance with immunohistochemical staining. The uPAR antibody was kindly provided by A. Mazar (Monopar).

Data Availability The datasets generated during and/or analyzed during the current study are available from the corresponding author on reasonable request.

\section{Compliance with Ethical Standards}

Funding No sources of funding were used to conduct this study or prepare this manuscript.

Conflict of interest DG, MR, TB, VB, CS, SH, GD, IN, BK, CR, and $\mathrm{RP}$ have no conflicts of interest that are directly relevant to the content of this article.

Ethical approval This study was performed according to the rules of the Medical Ethics Committee of the Radboud University Medical Center, registration number 2017-3759. All procedures performed in studies involving human participants were in accordance with the ethical standards of the institutional and/or national research committee and with the 1964 Helsinki declaration and its later amendments or comparable ethical standards. Formal consent is not required for this kind of study.

Open Access This article is licensed under a Creative Commons Attribution-NonCommercial 4.0 International License, which permits any non-commercial use, sharing, adaptation, distribution and reproduction in any medium or format, as long as you give appropriate credit to the original author(s) and the source, provide a link to the Creative Commons licence, and indicate if changes were made. The images or other third party material in this article are included in the article's Creative Commons licence, unless indicated otherwise in a credit line to the material. If material is not included in the article's Creative Commons licence and your intended use is not permitted by statutory regulation or exceeds the permitted use, you will need to obtain permission directly from the copyright holder.To view a copy of this licence, visit http://creativecommons.org/licenses/by-nc/4.0/.

\section{References}

1. Gupta B, Kumar N. Worldwide incidence, mortality and time trends for cancer of the oesophagus. Eur J Cancer Prev. 2017;26(2):107-18. https://doi.org/10.1097/cej.0000000000 000249 .

2. Dikken JL, Lemmens VE, Wouters MW, Wijnhoven BP, Siersema PD, Nieuwenhuijzen GA, et al. Increased incidence and survival for oesophageal cancer but not for gastric cardia cancer in the Netherlands. Eur J Cancer. 2012;48(11):1624-32. https://doi. org/10.1016/j.ejca.2012.01.009.

3. van Hagen P, Hulshof MC, van Lanschot JJ, Steyerberg EW, van Berge Henegouwen MI, Wijnhoven BP, et al. Preoperative chemoradiotherapy for esophageal or junctional cancer. N Engl J Med. 2012;366(22):2074-84. https://doi.org/10.1056/nejmo a1112088.

4. de Gouw DJJM, Scholte M, Gisbertz SS, Wijnhoven BPL, Rovers MM, Klarenbeek BR et al. The extent and consequences of lymphadenectomy in esophageal cancer surgery: a case vignette survey. 2019 (unpublished).

5. de Gouw D, Klarenbeek BR, Driessen M, Bouwense SAW, van Workum F, Futterer JJ, et al. Detecting pathological complete response in esophageal cancer after neoadjuvant therapy based on imaging techniques: a diagnostic systematic review and meta-analysis. J Thorac Oncol. 2019. https://doi.org/10.1016/j. jtho.2019.04.004.

6. Van Rossum PSN, Goense L, Meziani J, Reitsma JB, Siersema PD, Vleggaar FP, et al. Endoscopic biopsy and EUS for the detection of pathologic complete response after neoadjuvant chemoradiotherapy in esophageal cancer: a systematic review and meta-analysis. Gastrointest Endosc. 2016;83(5):866-79.

7. Keyaerts M, Xavier C, Heemskerk J, Devoogdt N, Everaert H, Ackaert C, et al. Phase I study of 68 Ga-HER2-Nanobody for PET/CT assessment of HER2-expression in breast carcinoma. J Nucl Med. 2015. https://doi.org/10.2967/jnumed.115.162024.

8. van Dam GM, Themelis G, Crane LM, Harlaar NJ, Pleijhuis RG, Kelder W, et al. Intraoperative tumor-specific fluorescence imaging in ovarian cancer by folate receptor-alpha targeting: first in-human results. Nat Med. 2011;17(10):1315-9. https:// doi.org/10.1038/nm.2472.

9. Wright BD, Lapi SE. Designing the magic bullet? The advancement of immuno-PET into clinical use. J Nucl Med. 2013;54(8):1171-4. https://doi.org/10.2967/jnumed.113.12608 6.

10. Hekman MCH, Rijpkema M, Aarntzen EH, Mulder SF, Langenhuijsen JF, Oosterwijk E, et al. Positron emission tomography/computed tomography with (89)Zr-girentuximab can aid in diagnostic dilemmas of clear cell renal cell carcinoma suspicion. Eur Urol. 2018;74(3):257-60. https://doi.org/10.1016/j.eururo.2018.04.026.

11. Rosenthal EL, Moore LS, Tipirneni K, de Boer E, Stevens TM, Hartman YE, et al. Sensitivity and specificity of cetuximabIRDye800CW to identify regional metastatic disease in head and neck cancer. Clin Cancer Res. 2017;23(16):4744-52. https://doi. org/10.1158/1078-0432.ccr-16-2968.

12. Lamberts LE, Koch M, de Jong JS, Adams ALL, Glatz J, Kranendonk MEG, et al. Tumor-specific uptake of fluorescent bevacizumab-IRDye $800 \mathrm{CW}$ microdosing in patients with primary breast cancer: a phase I feasibility study. Clin Cancer Res. 2017;23(11):2730-41. https://doi.org/10.1158/1078-0432. ccr-16-0437.

13. Hekman MC, Rijpkema M, Muselaers $\mathrm{CH}$, Oosterwijk E, de Kaa CA, Boerman OC, et al. Tumor-targeted dual-modality imaging to improve intraoperative visualization of clear cell renal cell 
carcinoma: a first in man study. Theranostics. 2018;8(8):2161-70. https://doi.org/10.7150/thno.23335.

14. Tang Z, Li C, Kang B, Gao G, Li C, Zhang Z. GEPIA: a web server for cancer and normal gene expression profiling and interactive analyses. Nucleic Acids Res. 2017;45(W1):W98-102. https ://doi.org/10.1093/nar/gkx247.

15. Sega EI, Low PS. Tumor detection using folate receptor-targeted imaging agents. Cancer Metastasis Rev. 2008;27(4):655-64. https ://doi.org/10.1007/s10555-008-9155-6.

16. Driessen A, Landuyt W, Pastorekova S, Moons J, Goethals L, Haustermans K, et al. Expression of carbonic anhydrase IX (CA IX), a hypoxia-related protein, rather than vascular-endothelial growth factor (VEGF), a pro-angiogenic factor, correlates with an extremely poor prognosis in esophageal and gastric adenocarcinomas. Ann Surg. 2006;243(3):334-40. https://doi.org/10.1097/01. sla.0000201452.09591.f3.

17. Alessi C, Scapulatempo Neto C, Viana CR, Vazquez VL. PD-1/PD-L1 and VEGF-A/VEGF-C expression in lymph node microenvironment and association with melanoma metastasis and survival. Melanoma Res. 2017;27(6):565-72. https://doi. org/10.1097/cmr.0000000000000396.

18. Chan E, et al. EGFR family and cMet expression profiles and prognostic significance in esophagogastric adenocarcinoma. JGastrointest Oncol. 2016;7(6):838-47.

19. Chan DS, Twine CP, Lewis WG. Systematic review and metaanalysis of the influence of HER2 expression and amplification in operable oesophageal cancer. J Gastrointest Surg. 2012;16(10):1821-9. https://doi.org/10.1007/s11605-012-1979-2.

20. Brungs D, Chen J, Aghmesheh M, Vine KL, Becker TM, Carolan MG, et al. The urokinase plasminogen activation system in gastroesophageal cancer: a systematic review and meta-analysis. Oncotarget. 2017;8(14):23099-109. https://doi.org/10.18632/ oncotarget.15485.

21. Christensen A, Kiss K, Lelkaitis G, Juhl K, Persson M, Charabi $\mathrm{BW}$, et al. Urokinase-type plasminogen activator receptor (uPAR), tissue factor (TF) and epidermal growth factor receptor (EGFR): tumor expression patterns and prognostic value in oral cancer. BMC Cancer. 2017;17(1):572. https://doi.org/10.1186/s1288 5-017-3563-3.

22. Adil Butt M, Pye H, Haidry RJ, Oukrif D, Khan SU, Puccio I, et al. Upregulation of mucin glycoprotein MUC1 in the progression to esophageal adenocarcinoma and therapeutic potential with a targeted photoactive antibody-drug conjugate. Oncotarget. 2017;8(15):25080-96. https://doi.org/10.18632/oncotarget.15340

23. Zhang J, Zhu Z, Liu Y, Jin X, Xu Z, Yu Q, et al. Diagnostic value of multiple tumor markers for patients with esophageal carcinoma. PLoS One. 2015;10(2):e0116951. https://doi.org/10.1371/journ al.pone.0116951.

24. Kimura O, Kondo Y, Kogure T, Kakazu E, Ninomiya M, Iwata T, et al. Expression of EpCAM increases in the hepatitis B related and the treatment-resistant hepatocellular carcinoma. Biomed Res Int. 2014;2014:172913. https://doi.org/10.1155/2014/172913.

25. Piessen G, Wacrenier A, Briez N, Triboulet JP, Van Seuningen I, Mariette C. Clinical impact of MUC1 and MUC4 expression in Barrett-associated oesophageal adenocarcinoma. J Clin Pathol. 2009;62(12):1144-6. https://doi.org/10.1136/jcp.2008.060780.
26. Taylor CR, Levenson RM. Quantification of immunohistochemistry-issues concerning methods, utility and semiquantitative assessment II. Histopathology. 2006;49(4):411-24. https://doi. org/10.1111/j.1365-2559.2006.02513.x.

27. Boogerd LSF, Hoogstins CES, Schaap DP, Kusters M, Handgraaf HJM, van der Valk MJM, et al. Safety and effectiveness of SGM101, a fluorescent antibody targeting carcinoembryonic antigen, for intraoperative detection of colorectal cancer: a dose-escalation pilot study. Lancet Gastroenterol Hepatol. 2018;3(3):181-91. https://doi.org/10.1016/s2468-1253(17)30395-3.

28. Hoogstins CES, Boogerd LSF, Sibinga Mulder BG, Mieog JSD, Swijnenburg RJ, van de Velde $\mathrm{CJH}$, et al. Image-guided surgery in patients with pancreatic cancer: first results of a clinical trial using SGM-101, a novel carcinoembryonic antigen-targeting, nearinfrared fluorescent agent. Ann Surg Oncol. 2018;25(11):3350-7. https://doi.org/10.1245/s10434-018-6655-7.

29. Boogerd LS, Boonstra MC, Prevoo HA, Handgraaf HJ, Kuppen $\mathrm{PJ}$, van de Velde CJ, et al. Fluorescence-guided tumor detection with a novel anti-EpCAM targeted antibody fragment: preclinical validation. Surg Oncol. 2019;28:1-8.

30. Bertero L, Massa F, Metovic J, Zanetti R, Castellano I, Ricardi U, et al. Eighth Edition of the UICC Classification of Malignant Tumours: an overview of the changes in the pathological TNM classification criteria-what has changed and why? Virchows Arch. 2018;472(4):519-31. https://doi.org/10.1007/s00428-017-2276-y.

31. Verlato G, Zanoni A, Tomezzoli A, Minicozzi A, Giacopuzzi S, Di Cosmo M, et al. Response to induction therapy in oesophageal and cardia carcinoma using Mandard tumour regression grade or size of residual foci. Br J Surg. 2010;97(5):719-25.

32. Burggraaf J, Kamerling IM, Gordon PB, Schrier L, de Kam ML, Kales AJ, et al. Detection of colorectal polyps in humans using an intravenously administered fluorescent peptide targeted against c-Met. Nat Med. 2015;21(8):955-61. https://doi.org/10.1038/ nm.3641.

33. Dadparvar S, Krishna L, Miyamoto C, Brady LW, Brown SJ, Bender $\mathrm{H}$, et al. Indium-111-labeled anti-EGFr-425 scintigraphy in the detection of malignant gliomas. Cancer. 1994;73(3 Suppl):884-9.

34. Hughes OD, Perkins AC, Frier M, Wastie ML, Denton G, Price MR, et al. Imaging for staging bladder cancer: a clinical study of intravenous 111indium-labelled anti-MUC1 mucin monoclonal antibody C595. BJU Int. 2001;87(1):39-46.

35. Dijkers EC, Oude Munnink TH, Kosterink JG, Brouwers AH, Jager PL, de Jong JR, et al. Biodistribution of 89Zr-trastuzumab and PET imaging of HER2-positive lesions in patients with metastatic breast cancer. Clin Pharmacol Ther. 2010;87(5):586-92. https://doi.org/10.1038/clpt.2010.12.

36. Skovgaard D, Persson M, Brandt-Larsen M, Christensen C, Madsen J, Klausen TL, et al. Safety, dosimetry, and tumor detection ability of (68)Ga-NOTA-AE105: first-in-human study of a novel radioligand for uPAR PET imaging. J Nucl Med. 2017;58(3):379_ 86. https://doi.org/10.2967/jnumed.116.178970.

37. Meyer JP, Edwards KJ, Kozlowski P, Backer MV, Backer JM, Lewis JS. Selective imaging of VEGFR-1 and VEGFR-2 using 89Zr-labeled single-chain VEGF mutants. J Nucl Med. 2016;57(11):1811-6. https://doi.org/10.2967/jnumed.116.173237. 


\section{Affiliations}

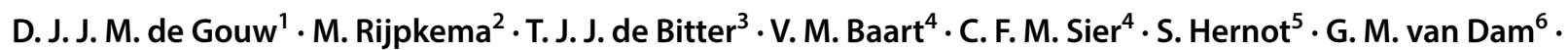
I. D. Nagtegaal ${ }^{3}$. B. R. Klarenbeek ${ }^{1}$. C. Rosman ${ }^{1}$ R. S. van der Post ${ }^{3}$

1 Department of Surgery, Radboud university medical center, Nijmegen, The Netherlands

2 Department of Radiology and Nuclear Medicine, Radboud university medical center, Nijmegen, The Netherlands

3 Department of Pathology, Radboud university medical center, Geert Grooteplein Zuid 30, 6525 GA Nijmegen, The Netherlands
4 Department of Surgery, Leiden University Medical Center, Leiden, The Netherlands

5 Laboratory for In vivo Cellular and Molecular Imaging, Vrije Universiteit Brussel, Brussel, Belgium

6 Department of Surgery and department of Nuclear Medicine and Medical Imaging Center, University Medical Center Groningen, Groningen, The Netherlands 